\title{
Analysis of the Trends in Publications on Clinical Cancer Research in Mainland China from the Surveillance, Epidemiology, and End Results (SEER) Database: Bibliometric Study
}

Min-Qiang Lin ${ }^{1 *}$, MSc; Chen-Lu Lian ${ }^{2 *}$, MD; Ping Zhou ${ }^{2 *}$, MD; Jian Lei ${ }^{3}$, MD; Jun Wang ${ }^{2}$, MD; Li Hua ${ }^{3}$, MD; Juan Zhou $^{3}$, MD; San-Gang $\mathrm{Wu}^{2}$, MD

\footnotetext{
${ }^{1}$ Department of Scientific Management, The First Affiliated Hospital of Xiamen University, Xiamen, China

${ }^{2}$ Department of Radiation Oncology, The First Affiliated Hospital of Xiamen University, Xiamen, China

${ }^{3}$ Department of Obstetrics and Gynecology, The First Affiliated Hospital of Xiamen University, Xiamen, China

these authors contributed equally
}

\section{Corresponding Author:}

San-Gang Wu, MD

Department of Radiation Oncology, The First Affiliated Hospital of Xiamen University

55 Zhenhai Road

Xiamen

China

Phone: 865922139531

Email: wusg@xmu.edu.cn

\begin{abstract}
Background: The application of China's big data sector in cancer research is just the beginning. In recent decades, more and more Chinese scholars have used the Surveillance, Epidemiology, and End Results (SEER) database for clinical cancer research. A comprehensive bibliometric study is required to analyze the tendency of Chinese scholars to utilize the SEER database for clinical cancer research and provide a reference for the future of big data analytics.
\end{abstract}

Objective: Our study aimed to assess the trend of publications on clinical cancer research in mainland China from the SEER database.

Methods: We performed a PubMed search to identify papers published with data from the SEER database in mainland China until August 31, 2020.

Results: A total of 1566 papers utilizing the SEER database that were authored by investigators in mainland China were identified. Over the past years, significant growth in studies based on the SEER database was observed $(P<.001)$. The top 5 research topics were breast cancer $(213 / 1566,13.6 \%)$, followed by colorectal cancer $(185 / 1566,11.8 \%)$, lung cancer $(179 / 1566$, $11.4 \%$ ), gastrointestinal cancer (excluding colorectal cancer; 149/1566, 9.5\%), and genital system cancer (93/1566, 5.9\%). Approximately $75.2 \%$ (1178/1566) of papers were published from the eastern coastal region of China, and Fudan University Shanghai Cancer Center (Shanghai, China) was the most active organization. Overall, 267 journals were analyzed in this study, of which Oncotarget was the most contributing journal (136/267, 50.9\%). Of the 1566 papers studied, 585 (37.4\%) were published in the second quartile, $489(31.2 \%)$ in the third quartile, $312(19.9 \%)$ in the first quartile, and $80(5.1 \%)$ in the fourth quartile, with 100 (6.4\%) having an unknown Journal Citation Reports ranking.

Conclusions: Clinical cancer research based on the SEER database in mainland China underwent constant and rapid growth during recent years. High-quality and comprehensive cancer databases based on Chinese demographic data are urgently needed.

(JMIR Med Inform 2020;8(11):e21931) doi: 10.2196/21931

\section{KEYWORDS}

cancer; China; data collection; bibliometrics; PubMed; SEER program 


\section{Introduction}

\section{Background}

The incidence of human cancer is increasing worldwide. It is estimated that the global burden of cancer will increase by more than $60 \%$ by 2040 [1]. Cancer has been an important public health problem in low- and middle-income countries, as well as in upper-middle-income countries. Cancer research has become one of the leading research fields of bioscience around the world, and the number of publications on cancer increases at a rate of more than $2 \%$ per year [2].

A high-quality cancer database can provide researchers with convenient data analysis and build a sharing platform among researchers, which could pave the way for revealing the mechanism of tumorigenesis and its progression [3]. To share clinical data with different regions, some other countries plunged into building multicenter databases far ahead of China [4]. In 1973, the US National Cancer Institute combined the tumor registration stations in several regions to form the Surveillance, Epidemiology, and End Results (SEER) database. The SEER program is a globally accessible authoritative cancer database representing approximately $34.6 \%$ of the US population, which includes non-Hispanic White, non-Hispanic Black, Hispanic, and Asian populations [5]. The SEER program collects data on patient demographics, tumor location, tumor stage, first course of therapy, and vital status. The SEER database is a valuable, population-based resource that can be used to study the diagnosis and treatment across demographic characteristics and geographic areas, and it has become a unique research resource for oncology practice. It provides morbidity and mortality data on various histopathological subtypes, and data on molecular characteristics are also expanding. The database is being further developed to capture other biomarker data and the results of specific populations, and to expand the biobank to support cutting-edge cancer research that can improve oncology practices. Therefore, the SEER program plays an important role in clinical cancer research, public health management, and policy making [5].

In recent years, China has made significant progress in clinical cancer research, and many studies have gained international recognition [6-10]. Although major hospitals have established databases in China, they have not shared their research findings with one another. Since the SEER database is a globally accessible authoritative cancer database, more and more scholars, particularly those from China, have used it to conduct clinical cancer research in recent years. There are a lot of differences between the United States and China, including their population's genetic makeup, health system, health services, health insurance, health policies, socioeconomic status, and culture. Therefore, the research findings in the SEER cancer registries may not be generalizable to the people of China. However, several recent studies found that the characteristics of the data from the SEER program were consistent with those from Chinese institutions [11-14]. There is currently no comprehensive bibliometric study that has characterized the clinical cancer research in China based on the SEER database. Carrying out a comprehensive bibliometric analysis is helpful to analyze the contribution of Chinese scholars to clinical cancer research and provide a specific clinical reference for the future of big data analytics.

\section{Objective}

This study aimed to evaluate the characteristics of clinical cancer research using SEER data from mainland China using a bibliometric approach.

\section{Methods}

\section{Search Strategy}

Using the search terms "Surveillance, Epidemiology, and End Results or SEER, and China," we identified related publications from the PubMed database before August 31, 2020. PubMed is a free, publicly available database established by the US National Library of Medicine [15]. As one of the largest databases in the life science and biomedical fields worldwide, it comprises more than 30 million biomedical abstracts from journals and online books.

Publications with first authors or corresponding authors affiliated with mainland Chinese institutions were included in the study, while those that utilized the SEER-Medicare database (SEER-Medicare data are not released outside of the United States) and special types of publications, including comments, letters, and reviews, were excluded. Papers from Taiwan Province, Hong Kong, and Macao Special Administrative Regions were also excluded from this analysis. This study was approved by the ethics committee of the First Affiliated Hospital of Xiamen University (Xiamen, Fujian, China).

\section{Indices}

The indices analyzed in this study included the number and trend of publications, research topics, type of affiliation (university, hospital, or other research center), geographical distribution, journal, Journal Citation Reports (JCR) ranking, and status of international cooperation.

\section{Statistical Analysis}

Descriptive statistical analyses were used in this study. Characteristics were evaluated and analyzed using Microsoft Excel, and linear regression was performed using SPSS statistical software (version 22.0; IBM Corp). $P$ values <.05 were considered statistically significant.

\section{Results}

\section{Number and Trend of Publications}

The flowchart of publication selection for this study is shown in Figure 1. We retrieved a total of 1667 publications, of which 1566 publications were included in this study. Figure 2 shows the trend in publications on clinical cancer research using the SEER database in mainland China $\left(R^{2}=0.430, P<.001\right)$. Chinese authors first used the SEER database in 1999. The single paper in the SEER database that was published in 1999 was authored by Guo et al [16], who were affiliated with the People's Hospital of Beijing Medical University and had collaborated with Huvos and colleagues from Memorial Sloan-Kettering Cancer Center 
in the United States. Interestingly, there were no papers from Chinese institutions that utilized the SEER database during the 11 years from 2000 to the end of 2011. However, the number of papers published per year increased rapidly from 2012 to
2019: $2012(\mathrm{n}=6), 2013(\mathrm{n}=12), 2014(\mathrm{n}=19), 2015 \quad(\mathrm{n}=43)$, $2016(n=96), 2017(n=181), 2018(n=288)$, and $2019(n=459)$. Despite the COVID-19 pandemic in 2020, there were 461 publications as of August 31, 2020.

Figure 1. Flowchart of publication selection for the study.

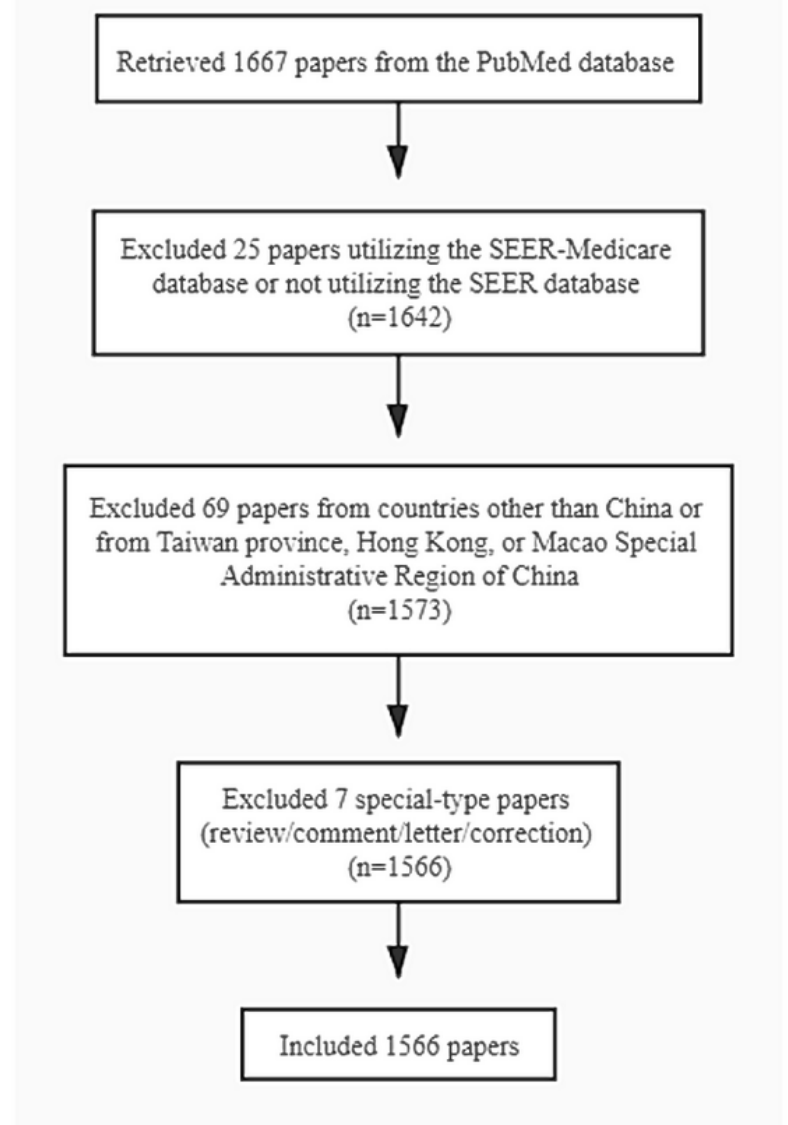

Figure 2. The trends in publications on clinical cancer research in mainland China from the Surveillance, Epidemiology, and End Results database between 1999 and 2020 .

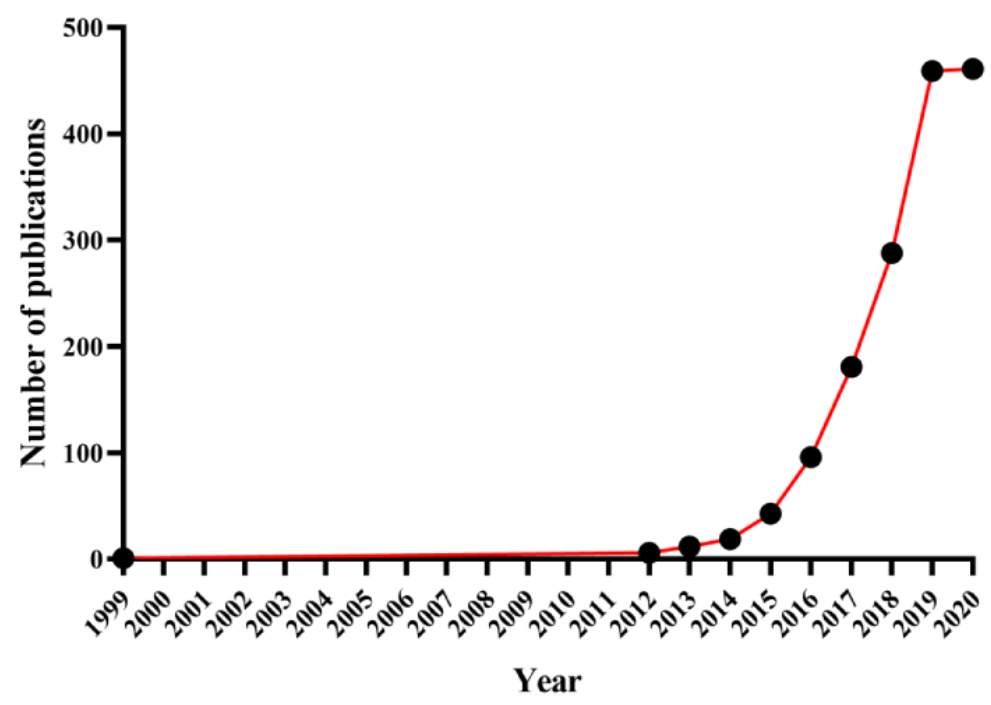

\section{Research Topics}

Table 1 presents the main research topics of the 1566 papers included in the study. Breast cancer was the most frequently researched topic $(213 / 1566,13.6 \%)$, followed by colorectal cancer $(185 / 1566,11.8 \%)$, lung cancer $(179 / 1566,11.4 \%)$, gastrointestinal cancer (excluding colorectal cancer; 149/1566, $9.5 \%)$, and genital system cancer $(93 / 1566,5.9 \%)$. These top 
5 research topics were the focus of $52.3 \%(819 / 1566)$ of the included papers.

Figure 3 shows the publication trends on the top 5 cancer sites in mainland China over time. It shows an increasing interest in research exploring breast cancer, lung cancer, gastrointestinal cancer (excluding colorectal cancer), colorectal cancer, and genital system cancer (in both genders). Chinese researchers were also studying rare cancers. A total of 164 papers discussed rare cancers, such as pulmonary lymphoepithelioma-like carcinoma, spindle cell carcinoma, thymoma, and adenoid cystic carcinoma of the breast, and this number was expanding rapidly in recent years.

Table 1. Main cancer research topics of publications in mainland China identified in the Surveillance, Epidemiology, and End Results database.

\begin{tabular}{ll}
\hline Main cancer research topics & Number of publications \\
\hline Breast cancer & 213 \\
Colorectal cancer & 185 \\
Lung cancer & 179 \\
Gastrointestinal cancer (excluding colorectal cancer) & 149 \\
Genital system cancer & 93 \\
Pancreatic cancer & 83 \\
Liver cancer & 72 \\
Thyroid cancer & 62 \\
Esophageal cancer & 62 \\
Bone cancer & 49 \\
\hline
\end{tabular}

Figure 3. The trends in publications on the top 5 cancer sites in mainland China from the Surveillance, Epidemiology, and End Results database between 2012 and 2020.

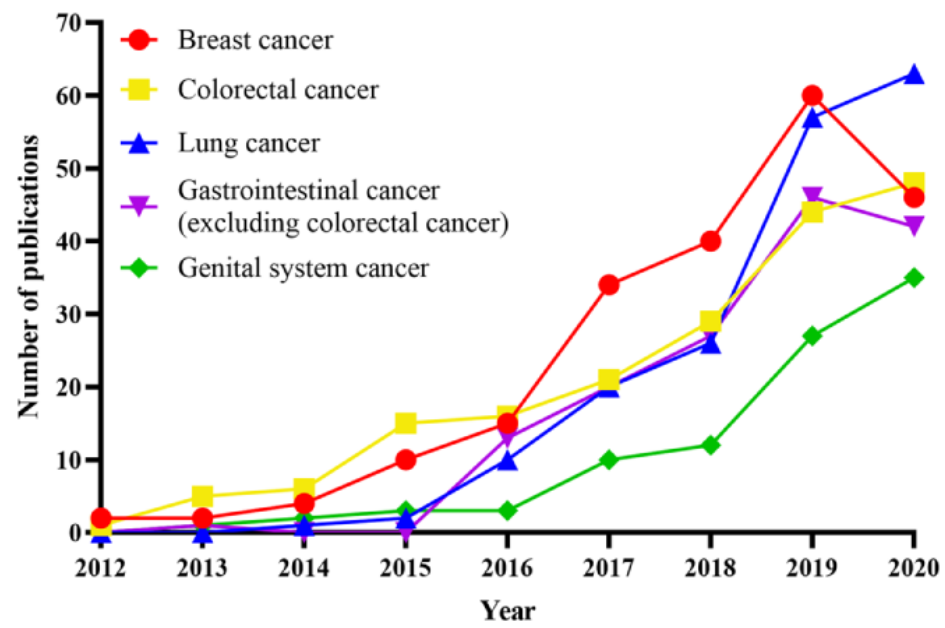

\section{Geographical Distribution of Publications}

Figure 4A shows the distribution of the publications using the SEER database whose first authors were affiliated with Chinese institutions. These first authors who used the SEER database to publish papers came from 25 provinces and municipalities of China. Among all of these regions, first authors were most often affiliated with institutions in Shanghai (369/1566, 23.6\%), followed by Guangdong (199/1566, 12.7\%), Zhejiang
(174/1566, 11.1\%), Hubei (102/1566, 6.5\%), and Jiangsu $(102 / 1566,6.5 \%)$.

Figure 4B shows the distribution of publications using the SEER database whose corresponding authors were affiliated with Chinese institutions. These corresponding authors who used the SEER database to publish papers came from 27 provinces or municipalities of China. A similar distribution was observed in the papers whose corresponding authors were affiliated with Chinese institutions, mostly concentrated in Shanghai, Guangdong, Zhejiang, Jiangsu, and Hubei. 
Figure 4. The distribution of the publications stratified by first authors (A) and corresponding authors (B) using the Surveillance, Epidemiology, and End Results database.

A

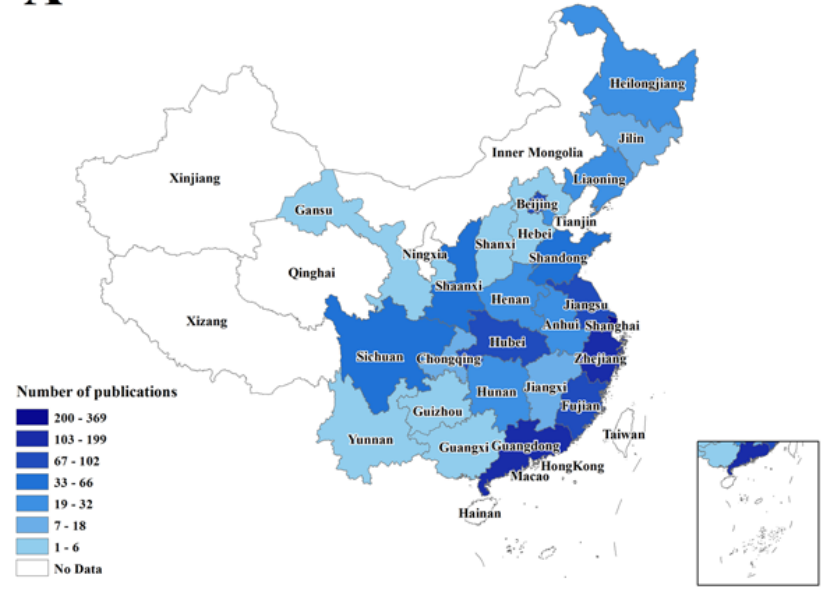

B

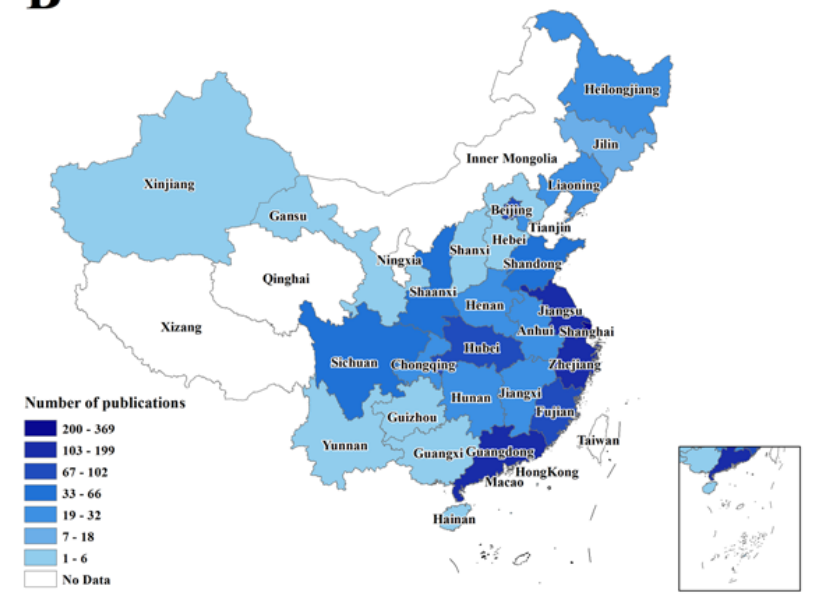

\section{Top 10 Most Contributing Author Affiliations}

The top 10 affiliated organizations of the most contributing first authors and corresponding authors extracted from the 1566 publications are presented in Tables 2 and 3. More than $75 \%$ (1178/1566) of papers were published from the eastern coastal region of China, Overall, the organization that was affiliated with the top contributing first authors was Fudan University Shanghai Cancer Center (163/1566, 10.4\%), followed by Sun Yat-sen University Cancer Center (73/1566, 4.7\%), the First Affiliated Hospital of Xi'an Jiaotong University (48/1566, $3.1 \%$ ), West China Hospital of Sichuan University (42/1566, $2.7 \%$ ), the Second Affiliated Hospital of Zhejiang University School of Medicine (41/1566, 2.6\%), and the First Affiliated Hospital of Xiamen University $(41 / 1566,2.6 \%)$. All affiliations of first authors extracted from the 1566 publications (excluding three papers whose first authors were affiliated with the United States) were classified into three sectors (university, hospital, and government agency). The most contributing institution sector was hospital $(1501 / 1563,96.0 \%)$, followed by university $(60 / 1563,3.8 \%)$, and government agency $(2 / 1563,0.1 \%)$. Of the 1501 papers from hospitals, 1459 (97.2\%) publications were produced from the best tertiary hospitals, and 1475 (98.3\%) were from hospitals affiliated with universities.

The organization that was affiliated with the top contributing corresponding authors was Fudan University Shanghai Cancer Center (165/1566, 10.5\%), followed by Sun Yat-sen University Cancer Center (77/1566, 4.9\%), Zhongshan Hospital of Fudan University $(43 / 1566,2.7 \%)$, the First Affiliated Hospital of Xi'an Jiaotong University (43/1566, 2.7\%), and the Second Affiliated Hospital of Zhejiang University School of Medicine (42/1566, 2.7\%). All affiliations of corresponding authors extracted from the 1566 papers (excluding 35 publications' organization affiliations of corresponding authors affiliated with the United States, Germany, Australia, and Japan) were also classified into three sectors (university, hospital, and government agency). Similarly, the most contributing institution sector was hospital $(1474 / 1531,96.3 \%)$, followed by university $(54 / 1531$, $3.5 \%)$, and government agency $(3 / 1531,0.2 \%)$. In the 1474 papers whose corresponding authors came from hospitals, 1440 (97.7\%) publications were from best tertiary hospitals, and 1458 $(98.9 \%)$ were from hospitals affiliated with universities.

Table 2. Top 10 affiliated organizations of the most contributing first authors.

\begin{tabular}{ll}
\hline Affiliations of first authors & Number of publications \\
\hline Fudan University Shanghai Cancer Center & 163 \\
Sun Yat-sen University Cancer Center & 73 \\
The First Affiliated Hospital of Xi' an Jiaotong University & 48 \\
West China Hospital of Sichuan University & 42 \\
The Second Affiliated Hospital of Zhejiang University & 41 \\
The First Affiliated Hospital of Xiamen University & 41 \\
Zhongshan Hospital of Fudan University & 39 \\
Affiliated Union Hospital of Fujian Medical University & 33 \\
Union Hospital of Tongji Medical College of Huazhong University of Science and Technology & 32 \\
Renmin Hospital of Wuhan University & 27 \\
\hline
\end{tabular}


Table 3. Top 10 affiliated organizations of the most contributing corresponding authors.

\begin{tabular}{ll}
\hline Affiliations of corresponding authors & Number of publications \\
\hline Fudan University Shanghai Cancer Center & 165 \\
Sun Yat-sen University Cancer Center & 77 \\
The First Affiliated Hospital of Xi' an Jiaotong University & 43 \\
Zhongshan Hospital of Fudan University & 43 \\
The Second Affiliated Hospital of Zhejiang University & 42 \\
West China Hospital of Sichuan University & 42 \\
Union Hospital of Tongji Medical College of Huazhong University of Science and Technology & 33 \\
Affiliated Union Hospital of Fujian Medical University & 31 \\
Renmin Hospital of Wuhan University & 27 \\
Shandong Cancer Hospital Affiliated to Shandong University & 27 \\
\hline
\end{tabular}

\section{Journals and Journal Visibility}

Overall, 267 journals were analyzed in the present study, of which the most contributing journal was Oncotarget (136/267, $50.9 \%$ ), followed by Cancer Medicine (98/267, 36.7\%), Journal of Cancer (76/267, 28.5\%), Medicine (Baltimore) (72/267, 27.0\%), and Cancer Management and Research (69/267, 25.8\%). The JCR ranking was retrieved from Web of Science, a global citation database. The top 30 most common journals extracted from the 1566 publications are shown in Figure 5. The distribution of the JCR ranking of the 1566 publications is depicted in Figure 6. A total of $37.4 \%$ (585/1566) of the publications were published in the second quartile, followed by the third quartile $(489 / 1566,31.2 \%)$, first quartile $(312 / 1566$, $19.9 \%)$, unknown JCR ranking (100/1566, 6.4\%), and fourth quartile $(80 / 1566,5.1 \%)$.

Figure 5. The distribution of the publications stratified by the top 30 most common journals.

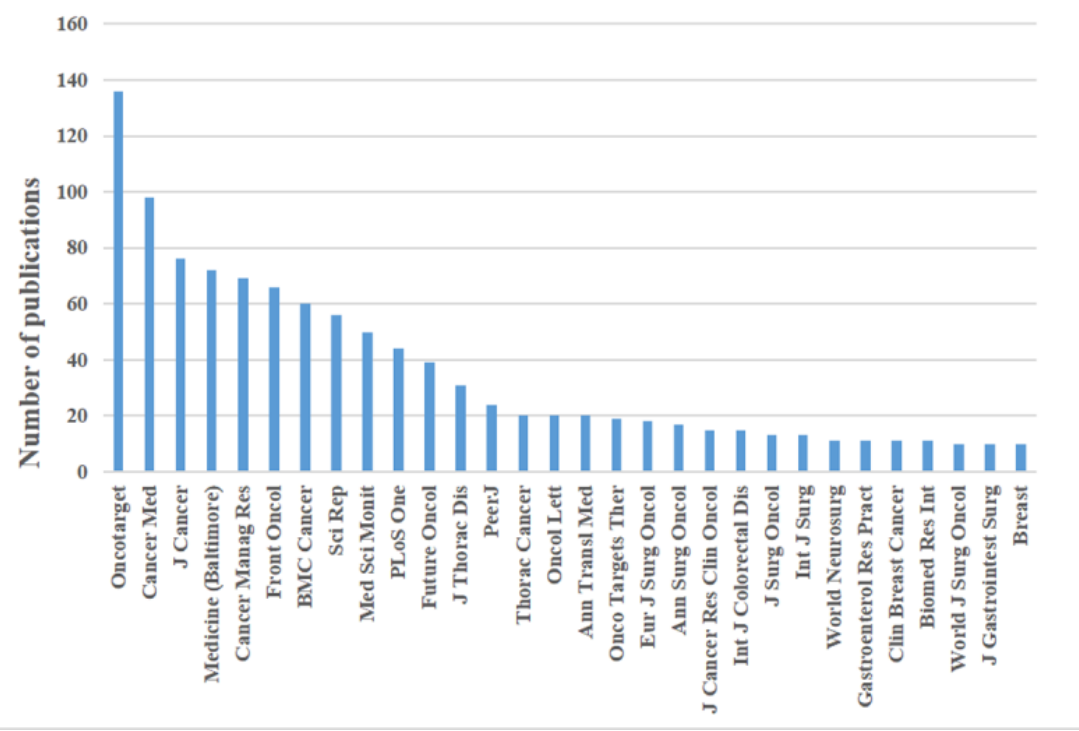


Figure 6. The distribution of the 1566 papers stratified by journal rankings published from Journal Citation Reports (JCR). Q1: first quartile; Q2: second quartile; Q3: third quartile; Q4: fourth quartile.

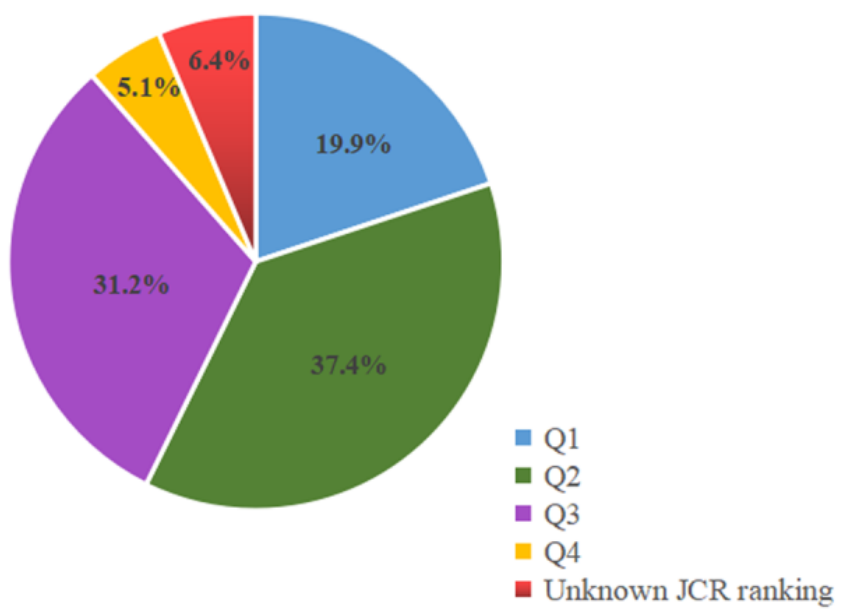

\section{International Cooperation}

A total of 180 publications were jointly produced by organizations from China and 23 other countries including the United States (114/180, 63.3\%), Russia (17/180, 9.4\%), Italy (12/180, 6.7\%), Australia (10/180, 5.6\%), and Japan (10/180, $5.6 \%)$. Chinese institutions first cooperated with institutions from other countries using the SEER database in 1999.
Similarly, no papers were jointly produced by institutions from China and other countries utilizing the SEER database during the 11 years from 2000 to the end of 2011 . However, the number of papers produced through international cooperation increased substantially from $2016(9 / 1566,0.6 \%)$ to $2019(65 / 1566,4.2 \%)$. As of August 31, 2020, a total of 45 papers had been published in the year 2020 with international cooperation (Figure 7).

Figure 7. The trends in publications with international cooperation using the Surveillance, Epidemiology, and End Results database between 1999 and 2020.

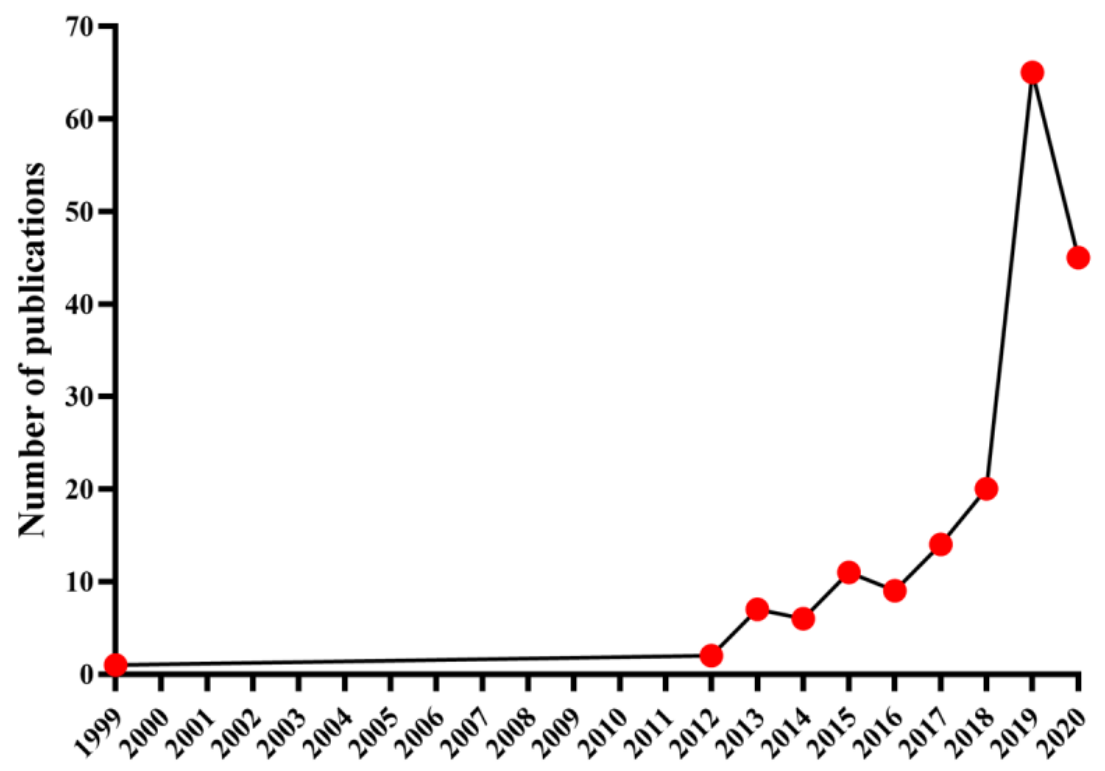

\section{Discussion}

The large sample size of the SEER database and strong statistical guarantees for current cancer concerns add much clinical value to research based on the SEER database. However, the application of China's big data sector in cancer research is just the beginning. In the recent decade, more and more Chinese scholars have used the SEER database for clinical cancer research. A comprehensive bibliometric study is required to analyze the tendency of Chinese scholars to utilize the SEER database for clinical cancer research and provide a reference for the future of big data analytics. In the present study, we completed a bibliometric study of the SEER database-related publications in China, some prominent characteristics of which were found. First, SEER database-related publications have made continuous and rapid growth in China in recent years. Second, the research topics mainly focused on high-incidence and high-mortality cancers, such as breast cancer, colorectal 
cancer, lung cancer, gastrointestinal cancer (excluding colorectal cancer), and genital system cancer. Third, publications regarding clinical cancer research using the data from the SEER program were mainly from the best tertiary hospitals in the eastern coastal region of China.

Our study revealed a rapidly growing interest in clinical cancer research from the SEER program in China. With the exception of 1 paper published in 1999, Chinese scholars did not utilize the SEER database for clinical cancer research until 2012. This trend has grown rapidly since 2012 , reaching 459 papers in the year 2019. The Covid-19 pandemic has changed almost every aspect of life and society in the year 2020. However, 461 publications were published in the first 8 months of 2020, indicating that the COVID-19 pandemic has not had a negative impact on publications in the SEER database. This phenomenon might attribute to the development of cancer research and informationization in China. Over the past decades, Chinese researchers have gradually made more in-depth use of cancer databases and noticed the important role of the SEER program. This finding was consistent with other studies that have demonstrated an increase in cancer research papers from China $[17,18]$. In addition, we noticed that not only Chinese scholars, but also scholars from France [19,20], South Korea [21,22], Japan [23], Italy [24], and Switzerland [25], have used the SEER database to conduct clinical cancer research.

With respect to the research topics, the SEER database-related publications were unbalanced, mainly concentrated on breast cancer, colorectal cancer, lung cancer, gastrointestinal cancer, and genital system cancer. Publications on these five cancer sites of the SEER database accounted for $52.3 \%$ of all 1566 publications. This regular pattern of distribution of the research topics was consistent with the top 5 cancer sites for estimated cases worldwide for both sexes, which were lung cancer, breast cancer, colorectal cancer, prostate cancer, and stomach cancer [1]. Although these top 5 research topics have been widely studied, Chinese researchers' interest in them has increased steadily. Moreover, the findings from the SEER studies have been introduced into the treatment guidelines of the National Comprehensive Cancer Network (NCCN) Guidelines and the European Society for Medical Oncology (ESMO) Guidelines, which demonstrate the critical role of the SEER database in clinical cancer research [26,27].

In addition to playing a significant role in the study of these high-incidence cancers, the SEER database is often used to explore rare diseases. Because rare cancers are very uncommon, it is difficult to collect data from a single institution. The SEER database provided substantial valuable data for the study of rare cancers and avoided the selection bias found in single-center retrospective studies. Nevertheless, the results from the SEER database are also retrospective, and prospective studies are needed to validate the results from the SEER database. However, rare cancers are challenging to study prospectively because of their low incidence. The latest NCCN and ESMO guidelines cited several publications on rare cancers based on SEER data, such as male breast cancer, occult breast cancer, and mesenchymal chondrosarcoma [28-30]. Thus, these publications based on SEER data provided insight to help clarify the characteristics, treatment protocols, prognostic indicators, and risk stratification of rare cancers $[31,32]$.

With respect to geographical and institutional distribution, papers published from China using the SEER database were extremely unbalanced. More than $75 \%$ of papers were published from the eastern coastal region of China, which was the area with the highest incidence of cancer morbidity in mainland China [33]. In our analysis, more than $90 \%$ of publications were produced by the best tertiary hospitals, while 4 of the top 5 contributing organizations were located in the eastern coastal region of China. Regarding the population and economic levels, high-quality medical resources are distributed unevenly in China, with 71 of the 2018 top 100 hospitals in China situated in the eastern coastal region of China [34-37]. In addition, most medical colleges in China are located in this region and could provide resources for cancer research [38]. By relying on universities, researchers at affiliated hospitals can get more research support.

The findings from the SEER program may contribute to cancer prevention and treatment, whether the cancer is common or rare. Nevertheless, the SEER program still has its limitations in that its vital statistics only consist of death and survival data, and there are no data regarding locoregional recurrence and distant metastasis after adopting the corresponding treatment. In addition, the SEER database only represents people who live in the United States. Whether the results of SEER-based research can be applied to other countries, especially to areas of high-incidence cancers like China, needs to be verified by data from other countries. Some Chinese researchers combined the SEER database with their databases to conduct studies and found that the characteristics of the data from the SEER program were consistent with that from Chinese institutions [11-14]. This indicated that the SEER database could provide valuable data for clinical oncology treatment in China. The above results indicate that clinical cancer research based on the SEER database may provide a valuable reference for clinical cancer researchers in China.

China should establish cancer databases based on its demographic characteristics. To our knowledge, Chinese cancer registration work was started much later than in other upper middle-income countries [4]. The number of cancer registries in China has increased rapidly since 2002, while a significant gap still exists between China and other upper middle-income countries. At present, the items and contents of population-based cancer data in China are limited. Therefore, high-quality cancer databases based on Chinese demographic data are urgently needed to better reflect the oncology practices in China [39-42].

The limitation of our analysis is that we only collected publications from the PubMed database, which may ignore some publications that were not indexed in PubMed, resulting in incomplete data.

\section{Conclusions}

In conclusion, our study suggests that clinical cancer research regarding the SEER database has rapidly increased in China in the past decade. High-quality and national comprehensive cancer 
registries from China are needed to provide a reference for the future of big data analytics.

\section{Authors' Contributions}

MQL, CLL, PZ, and SGW were responsible for the study design; MQL, CLL, PZ, and SGW contributed to writing the report; and JW, JL, LH, and JZ contributed to data analysis. All authors have reviewed and approved the final manuscript.

\section{Conflicts of Interest}

None declared.

\section{References}

1. Bray F, Ferlay J, Soerjomataram I, Siegel RL, Torre LA, Jemal A. Global cancer statistics 2018: GLOBOCAN estimates of incidence and mortality worldwide for 36 cancers in 185 countries. CA Cancer J Clin 2018 Nov;68(6):394-424 [FREE Full text] [doi: 10.3322/caac.21492] [Medline: $\underline{30207593}$ ]

2. Lewison G, Purushotham A, Mason M, McVie G, Sullivan R. Understanding the impact of public policy on cancer research: a bibliometric approach. Eur J Cancer 2010 Mar;46(5):912-919. [doi: 10.1016/j.ejca.2009.12.020] [Medline: 20064708]

3. Yang J, Cai HY. [The Cancer-related Bioinformatics Databases] [In Chinese]. Biotechnology Bulletin 2015 Nov;31(11):89-101. [doi: 10.13560/j.cnki.biotech.bull.1985.2015.11.010]

4. Yang L. [Brief Review on Cancer Registration at Home and Abroad] [In Chinese]. China Cancer 2005 Dec;14(12):772-775. [doi: 10.3969/j.issn.1004-0242.2005.12.001]

5. The Surveillance, Epidemiology, and End Results (SEER) Program Overview. National Cancer Institute. URL: https://seer. cancer.gov/about/overview.html [accessed 2019-12-20] [WebCite Cache ID https://seer.cancer.gov/about/overview.html]

6. Zhang Y, Chen L, Hu GQ, Zhang N, Zhu XD, Yang KY, et al. Gemcitabine and Cisplatin Induction Chemotherapy in Nasopharyngeal Carcinoma. N Engl J Med 2019 Sep 19;381(12):1124-1135. [doi: 10.1056/NEJMoa1905287] [Medline: 31150573]

7. Chen Y, Ye J, Zhu Z, Zhao W, Zhou J, Wu C, et al. Comparing Paclitaxel Plus Fluorouracil Versus Cisplatin Plus Fluorouracil in Chemoradiotherapy for Locally Advanced Esophageal Squamous Cell Cancer: A Randomized, Multicenter, Phase III Clinical Trial. J Clin Oncol 2019 Jul 10;37(20):1695-1703 [FREE Full text] [doi: 10.1200/JCO.18.02122] [Medline: 30920880]

8. Ma F, Ouyang Q, Li W, Jiang Z, Tong Z, Liu Y, et al. Pyrotinib or Lapatinib Combined With Capecitabine in HER2-Positive Metastatic Breast Cancer With Prior Taxanes, Anthracyclines, and/or Trastuzumab: A Randomized, Phase II Study. J Clin Oncol 2019 Oct 10;37(29):2610-2619. [doi: 10.1200/JCO.19.00108] [Medline: $\underline{\text { 31430226] }}$

9. Luo Y, Luo L, Wampfler JA, Wang Y, Liu D, Chen Y, et al. 5-year overall survival in patients with lung cancer eligible or ineligible for screening according to US Preventive Services Task Force criteria: a prospective, observational cohort study. Lancet Oncol 2019 Aug;20(8):1098-1108 [FREE Full text] [doi: 10.1016/S1470-2045(19)30329-8] [Medline: $\underline{31255490]}$

10. $\mathrm{Li} \mathrm{Y,} \mathrm{Wu} \mathrm{YL.} \mathrm{The} \mathrm{second} \mathrm{wave} \mathrm{of} \mathrm{checkpoint} \mathrm{inhibitors} \mathrm{with} \mathrm{chemotherapy} \mathrm{for} \mathrm{advanced} \mathrm{non-small-cell} \mathrm{lung} \mathrm{cancer.}$ Lancet Oncol 2019 Jul;20(7):889-891. [doi: 10.1016/S1470-2045(19)30148-2] [Medline: 31122902]

11. Hua J, Zhang B, Xu J, Liu J, Ni Q, He J, et al. Determining the optimal number of examined lymph nodes for accurate staging of pancreatic cancer: An analysis using the nodal staging score model. Eur J Surg Oncol 2019 Jun;45(6):1069-1076 [FREE Full text] [doi: 10.1016/j.ejso.2019.01.018] [Medline: $\underline{30685327]}$

12. Shi X, Liu XK, An CM, Wei WJ, Tao Y, Ji Y, et al. Anatomic extent of lymph node metastases as an independent prognosticator in node-positive major salivary gland carcinoma: A study of the US SEER database and a Chinese multicenter cohort. Eur J Surg Oncol 2019 Nov;45(11):2143-2150. [doi: 10.1016/j.ejso.2019.06.029] [Medline: 31253544]

13. He J, Tsang JY, Xu X, Li J, Li M, Chao X, et al. AJCC 8th edition prognostic staging provides no better discriminatory ability in prognosis than anatomical staging in triple negative breast cancer. BMC Cancer 2020 Jan 06;20(1):18 [FREE Full text] [doi: 10.1186/s12885-019-6494-3] [Medline: 31906874]

14. Liang W, He J, Shen Y, Shen J, He Q, Zhang J, et al. Impact of Examined Lymph Node Count on Precise Staging and Long-Term Survival of Resected Non-Small-Cell Lung Cancer: A Population Study of the US SEER Database and a Chinese Multi-Institutional Registry. J Clin Oncol 2017 Apr 10;35(11):1162-1170 [FREE Full text] [doi: 10.1200/JCO.2016.67.5140] [Medline: 28029318]

15. PubMed. URL: https://www.ncbi.nlm.nih.gov/pubmed/ [accessed 2019-12-20]

16. Guo W, Xu W, Huvos AG, Healey JH, Feng C. Comparative frequency of bone sarcomas among different racial groups. Chin Med J (Engl) 1999 Dec;112(12):1101-1104. [Medline: 11721448]

17. Zhang LP. [Oncology literature in China from 1994 to 2003: a bibliometric analysis] [In Chinese]. Chin J Med Libr Inf Sci 2006 Jan;15(1):67-69.

18. Zheng JJ, Zhang HR, Jing S. [A bibliometric analysis of oncology papers published by Chinese authors covered in SCI from 2010 to 2012] [In Chinese]. Chin J Med Libr Inf Sci 2012 Dec;21(12):64-71. [doi:

10.3969/j.issn.1671-3982.2012.12.022] 
19. Benoit L, Pauly L, Phelippeau J, Koskas M. Impact of Sociodemographic Characteristics on the Quality of Care in the Surgical Management of Endometrial Cancer: An Analysis of a National Database in the United States. Gynecol Obstet Invest 2020 Mar;85(3):222-228. [doi: 10.1159/000506048] [Medline: $\underline{\text { 32224609] }}$

20. Gonthier C, Douhnai D, Koskas M. Lymph node metastasis probability in young patients eligible for conservative management of endometrial cancer. Gynecol Oncol 2020 Apr;157(1):131-135. [doi: 10.1016/j.ygyno.2020.02.021] [Medline: 32139150]

21. Kim BH, Kim S, Kim YI, Chang JH, Hwang K, Kim S, et al. Development of an Individualized Prediction Calculator for the Benefit of Postoperative Radiotherapy in Patients with Surgically Resected De Novo Stage IV Breast Cancer. Cancers (Basel) 2020 Jul 29;12(8):2103 [FREE Full text] [doi: 10.3390/cancers12082103] [Medline: $\underline{32751136]}$

22. Jung J, Kim BH, Kim J, Oh S, Kim SJ, Lim CS, et al. Validating the ACOSOG Z0011 Trial Result: A Population-Based Study Using the SEER Database. Cancers (Basel) 2020 Apr 11;12(4):950 [FREE Full text] [doi: 10.3390/cancers12040950] [Medline: 32290437]

23. Watanuki R, Hayashida T, Yokoe T, Kawai Y, Kikuchi M, Nakashoji A, et al. Impact of neoadjuvant and adjuvant chemotherapy on invasive lobular carcinoma: A propensity score-matched analysis of SEER data. Breast J 2020 May 25 (forthcoming). [doi: 10.1111/tbj.13884] [Medline: $\underline{\text { 32449173] }}$

24. Rosiello G, Palumbo C, Pecoraro A, Luzzago S, Deuker M, Stolzenbach LF, et al. The effect of sex on disease stage and survival after radical cystectomy: a population-based analysis. Urol Oncol 2020 Oct 06 (forthcoming). [doi: 10.1016/j.urolonc.2020.09.004] [Medline: 33036900]

25. Siebenhüner AR, Güller U, Warschkow R. Population-based SEER analysis of survival in colorectal cancer patients with or without resection of lung and liver metastases. BMC Cancer 2020 Mar;20(1):246. [doi: 10.1186/s12885-020-6710-1] [Medline: 32293337$]$

26. Petkov VI, Miller DP, Howlader N, Gliner N, Howe W, Schussler N, et al. Breast-cancer-specific mortality in patients treated based on the 21-gene assay: a SEER population-based study. NPJ Breast Cancer 2016 Jun;2:16017. [doi: 10.1038/npjbcancer.2016.17] [Medline: 28721379]

27. Qiu M, Hu J, Yang D, Cosgrove DP, Xu R. Pattern of distant metastases in colorectal cancer: a SEER based study. Oncotarget 2015 Nov;6(36):38658-38666. [doi: 10.18632/oncotarget.6130] [Medline: 26484417]

28. Cloyd JM, Hernandez-Boussard T, Wapnir IL. Outcomes of partial mastectomy in male breast cancer patients: analysis of SEER, 1983-2009. Ann Surg Oncol 2013 May;20(5):1545-1550. [doi: 10.1245/s10434-013-2918-5] [Medline: 23460016]

29. Wu SG, Zhang WW, Sun JY, Li FY, Lin HX, Chen YX, et al. Comparable Survival between Additional Radiotherapy and Local Surgery in Occult Breast Cancer after Axillary Lymph Node Dissection: A Population-based Analysis. J Cancer 2017 Oct;8(18):3849-3855. [doi: 10.7150/jca.21217] [Medline: 29151972]

30. Schneiderman BA, Kliethermes SA, Nystrom LM. Survival in Mesenchymal Chondrosarcoma Varies Based on Age and Tumor Location: A Survival Analysis of the SEER Database. Clin Orthop Relat Res 2017 Mar;475(3):799-805 [FREE Full text] [doi: 10.1007/s11999-016-4779-2] [Medline: 26975384]

31. Zhuo M, Zheng Q, Chi Y, Jia B, Zhao J, Wu M, et al. Survival analysis via nomogram of surgical patients with malignant pleural mesothelioma in the Surveillance, Epidemiology, and End Results database. Thorac Cancer 2019 May;10(5):1193-1202 [FREE Full text] [doi: 10.1111/1759-7714.13063] [Medline: 30951250]

32. Yin Z, Wang Y, Wu Y, Zhang X, Wang F, Wang P, et al. Age distribution and age-related outcomes of olfactory neuroblastoma: a population-based analysis. Cancer Manag Res 2018 May;10:1359-1364 [FREE Full text] [doi: 10.2147/CMAR.S151945] [Medline: 29881306]

33. Zheng RS, Sun KX, Zhang SW, Zeng HM, Zou XN, Chen R, et al. [Report of cancer epidemiology in China, 2015] [In Chinese]. Zhonghua Zhong Liu Za Zhi 2019 Jan 23;41(1):19-28. [doi: 10.3760/cma.j.issn.0253-3766.2019.01.005] [Medline: $\underline{30678413]}$

34. Yue LR, Sun LQ, Yang K, Li Z. [Research on the Status Quo and Strategy of China's Medical and Health System Reform under the Background of Reform and Opening-up] [In Chinese]. China Health Industry 2019 Jul;16(19):185-188. [doi: 10.16659/j.cnki.1672-5654.2019.19.185]

35. Shi BG, Wu SL. [Spatial Analysis of Access to Care:Geographic Distribution of China's Top Hospitals from The Perspective of Embedded Stratification Theory] [In Chinese]. Journal of Gansu Administration Institute 2019 Oct;5:94-104.

36. An YF. [Characters and Improvement Strategies of Distribution of High-Quality Medical Resources] [In Chinese]. Chinese Health Quality Management 2011 Sep;18(5):110-113. [doi: 10.13912/j.cnki.chqm.2011.05.034]

37. Zhuang YQ, Liao XB, Wang XL, Yao SF. [Hospital Blue Book: China Hospital Competitiveness Report (2018-2019)] [In Chinese]. Beijing: Social Sciences Academic Press (China); 2019.

38. ShanghaiRanking. URL: https://www.shanghairanking.com.cn/ [accessed 2019-12-20]

39. Wei WQ, He J. [Some thoughts on cancer registry in China: in the era of big data and informatization] [In Chinese]. Zhonghua Zhong Liu Za Zhi 2019 Jan 23;41(1):15-18. [doi: 10.3760/cma.j.issn.0253-3766.2019.01.004] [Medline: 30678412]

40. Zhang SW, Chen WQ, Wang L. [The 30 Years of Cancer Registration in China] [In Chinese]. China Cancer 2009 Apr;18(4):256-259. [doi: 10.3969/j.issn.1004-0242.2007.07.001]

41. Chen W. [Establishing and Perfecting of Cancer Registration System in China] [In Chinese]. China Cancer 2011 Jan;20(1):7-9.

42. Chen W, Zheng R, Baade PD, Zhang S, Zeng H, Bray F, et al. Cancer statistics in China, 2015. CA Cancer J Clin 2016 Mar;66(2):115-132 [FREE Full text] [doi: 10.3322/caac.21338] [Medline: 26808342] 


\title{
Abbreviations
}

ESMO: European Society for Medical Oncology

JCR: Journal Citation Reports

NCCN: National Comprehensive Cancer Network

SEER: Surveillance, Epidemiology, and End Results

\author{
Edited by C Lovis; submitted 29.06.20; peer-reviewed by P Yang, S Song; comments to author 06.09.20; revised version received \\ 19.10.20; accepted 25.10.20; published 17.11.20 \\ Please cite as: \\ Lin MQ, Lian CL, Zhou P, Lei J, Wang J, Hua L, Zhou J, Wu SG \\ Analysis of the Trends in Publications on Clinical Cancer Research in Mainland China from the Surveillance, Epidemiology, and \\ End Results (SEER) Database: Bibliometric Study \\ JMIR Med Inform 2020;8(11):e21931 \\ URL: http://medinform.jmir.org/2020/11/e21931/ \\ doi: $\underline{10.2196 / 21931}$ \\ PMID: 33200992
}

CMin-Qiang Lin, Chen-Lu Lian, Ping Zhou, Jian Lei, Jun Wang, Li Hua, Juan Zhou, San-Gang Wu. Originally published in JMIR Medical Informatics (http://medinform.jmir.org), 17.11.2020. This is an open-access article distributed under the terms of the Creative Commons Attribution License (https://creativecommons.org/licenses/by/4.0/), which permits unrestricted use, distribution, and reproduction in any medium, provided the original work, first published in JMIR Medical Informatics, is properly cited. The complete bibliographic information, a link to the original publication on http://medinform.jmir.org/, as well as this copyright and license information must be included. 